\title{
Théorie et instrumentation des techniques de LC-MS et LC-MS/MS applicables à la toxicologie
}

\section{$L C-M S$ and $L C-M S / M S$ theory and instruments applicable to toxicology}

Pierre MARQUET

Service de Pharmacologie-Toxicologie, CHU Dupuytren - 87042 LIMOGES Cedex - FRANCE Tél : 0555056140 - Fax : 0555056162 - E-mail : marquet@unilim.fr

(Reçu le 3 janvier 2005 ; accepté après modifications le 23 mars 2005)

\section{$R \hat{E} S U M E ́$}

Cet article passe en revue les principaux constituants d'un spectromètre de masse couplé à la chromatographie liquide (sources d'ionisation à pression atmosphérique, cellules de collision, filtres de masse) et, pour chacun, les principales innovations des constructeurs, leurs avantages proclamés, leur intérêt et leurs applications potentielles en toxicologie. Sont également abordés les modes opératoires généralement utilisés pour les analyses qualitatives ou quantitatives en toxicologie, ainsi que les rares recommandations internationales concernant le nombre d'ions ou de transitions de masse à enregistrer et les variations acceptables de leurs intensités relatives, permettant de profiter réellement de la spécificité de la LC-MS et de la LC-MS/MS au cours d'analyses quantitatives. Il est rappelé à cette occasion que des analyses toxicologiques fiables reposent non seulement sur un matériel performant, mais aussi sur de bonnes pratiques techniques et des opérateurs compétents.

\section{MOTS-CLÉS}

Sources d'ionisation, cellules de collision, filtres de masse, bonnes pratiques de laboratoire.

\section{SUMMARY}

This paper reviews the main parts of a mass spectrometer coupled to liquid chromatography (atmospheric pressure ionisation sources, collision cells, mass filters) and, for each, the main manufacturers' innovations, their claimed advantages and their potential interest and applications in toxicology.

The operating procedures most frequently used in toxicology for either qualitative or quantitative analysis are also discussed, as well as the rare international recommendations about the number of ions or mass transitions to monitor and the acceptable variability of their relative intensity, in order to fully benefit from LC-MS or LC-MS/MS specificity during quantitative assays. On this occasion, it is reminded that reliable toxicology analyses not only rely on high-performance devices, but also on good technical practices and skilled operators.

\section{KEY-WORDS}

Ionisation sources, collision cells, mass filters, good laboratory practices 


\section{Introduction}

Le couplage entre la chromatographie liquide (LC) haute performance (ou haute pression) et la spectrométrie de masse (MS, nécessitant un vide poussé) a toujours été le maillon faible de la LC-MS, qui permet théoriquement d'allier la quasi universalité de l'une et de l'autre. Il s'agit, en effet, de résoudre deux problèmes majeurs : éliminer de larges volumes de gaz et de vapeur produits par la phase mobile et transformer les molécules en solution dans la phase mobile en ions en suspension dans une phase gazeuse, sans dégradation thermique (1). Les sources d'ionisation ou interfaces de couplage historiques, telles que particle beam, thermospray ou moving-belt ayant été abandonnées, nous nous intéresserons ici aux principes et aux évolutions des sources d'ionisation à pression atmosphérique.

Parallèlement à l'histoire naturelle de ces interfaces, différents types de filtres de masse ont été progressivement développés, puis certains ont connu une meilleure fortune que d'autres. Les filtres de masse quadripolaires ont été très largement diffusés et utilisés, en particulier en toxicologie, du fait probablement de leur faible coût. Les couplages de la $\mathrm{LC}$ avec des systèmes à triple quadrupole, dits « en tandem » (LC-MS/MS) représentent aujourd'hui les plus fortes ventes dans l'industrie pharmaceutique (en particulier pour les études de toxicocinétique et de pharmacocinétique), ainsi probablement que dans les laboratoires de toxicologie médicale ou de suivi thérapeutique pharmacologique. Toutefois, avec l'avènement de la protéomique, on voit actuellement fleurir ou refleurir d'autres types de filtres de masse, de plus grande résolution, potentiellement applicables à la toxicologie (en particulier à l'identification de composés inconnus).

Cet article a pour but de présenter les principales technologies de LC-MS et LC-MS/MS existantes et de rappeler leur mode d'utilisation habituel ou recommandé en toxicologie.

\section{Les sources d'ionisation à pression atmosphérique}

Bien que leur description date de la fin des années 50 , les premières applications en toxicologie des sources à pression atmosphérique datent du début des années 90 (2). Les applications plus récentes ont fait l'objet de plusieurs articles de revue de littérature ces dernières années (3-6). Les interfaces à pression atmosphérique regroupent différentes versions de sources : electrospray, APCI (Atmospheric Pressure Chemical Ionisation) et, plus récemment, des sources de photo- ionisation ou APPI (Atmospheric Pressure PhotoIonisation). Leur caractéristique commune est la combinaison d'un système d'introduction du liquide, d'une source à pression atmosphérique, d'un orifice d'entrée des ions dans le spectromètre et d'une interface entre la pression atmosphérique et le vide poussé du spectromètre de masse, chacun de ces éléments présentant des spécifications propres à chaque fabricant.

Ces interfaces à pression atmosphérique et les sources d'ionisation correspondantes permettent de couvrir une très large gamme de polarité et de masse moléculaire : les interfaces electrospray permettent la détection de molécules polaires à très polaires, jusqu'à des masses moléculaires de plusieurs milliers de daltons (peptides, protéines) ; la source APCI permet la détection de molécules relativement apolaires et la source APPI la détection de molécules encore plus apolaires (figure 1). Ces interfaces sont généralement facilement interchangeables sur un même instrument, comme par exemple sur le système LC-MSD 1100 Series Agilent (7), ou combinés en une seule source comme la «Dual-source» de la société Applied Biosystems-Sciex (8), combinant les modes d'ionisation electrospray et APCI, permettant ainsi un passage quasi instantané de l'un à l'autre au cours d'une acquisition.

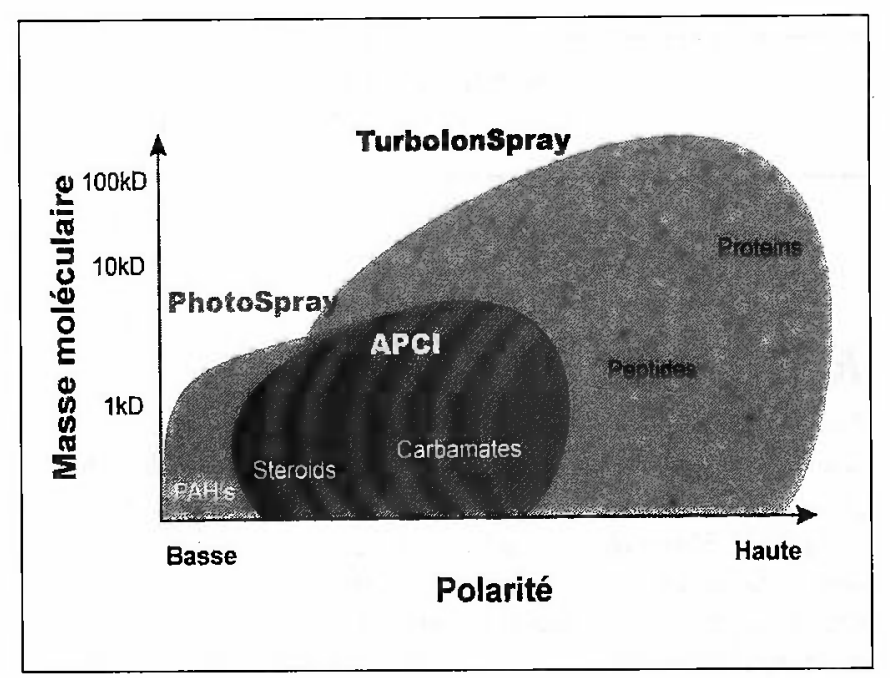

Figure 1 : Domaines de polarité et de masse moléculaire compatibles avec les différents types de sources à pression atmosphériques [d'après (8)]. PAH's : hydrocarbures polyaromatiques; steroids : stéroïdes ; proteins : protéines.

\section{Les sources de type electrospray pneu- matiquement assistées}

La nébulisation et l'ionisation de la phase mobile chromatographique sont effectuées simultanément, par application d'un champ électrique intense (plusieurs milliers de volts) entre l'aiguille de nébulisation et le 
corps de la source. L'axe de l'aiguille de nébulisation est généralement décentré par rapport à l'orifice du spectromètre de masse, de façon à éviter le passage de liquide ou de molécules neutres dans le vide de l'appareil. Certains constructeurs ont ainsi proposé des sources orthogonales, dans lesquelles le spray et l'axe du spectromètre de masse sont à angle droit. La désolvatation des ions dans la source est assistée par un courant de gaz (air ou azote), concentrique à l'effluent liquide, ainsi parfois que par un courant de gaz (éventuellement chauffé) perpendiculaire au spray. Les gouttelettes de phase mobile ionisée s'évaporent progressivement pendant le trajet dans la source, conduisant à une augmentation de la densité des charges à leur surface. Les forces électrostatiques de surface finissent par les faire exploser, libérant des ions désolvatés, positifs ou négatifs, éventuellement polyatomiques (clusters) (6). Les ions d'une seule polarité (positifs ou négatifs) sont extraits du spray par un champ électrique dirigé vers le spectromètre de masse. Chez certains constructeurs, un rideau de gaz sépare la source du spectromètre de masse, permettant d'éviter l'entrée de molécules neutres ou d'ions parasites, ainsi que de fragmenter les ions polyatomiques (processus de « declusterisation ») ce qui présente l'avantage de favoriser les molécules protonées plutôt que les adduits sodium, potassium ou ammonium, classiques dans le mode d'ionisation positif. Ce gaz rideau peut également participer au processus de fragmentation induite dans la source, utilisé pour générer des fragments qui serviront à confirmer l'identité de l'espèce chimique à analyser. Cette fragmentation s'opère essentiellement dans la zone de pression intermédiaire entre la source et le vide primaire, par accélération des ions à l'aide d'une tension électrique afin de provoquer des collisions avec les molécules de gaz résiduelles. Les systèmes électroniques utilisés par tous les constructeurs permettent à l'heure actuelle de faire varier et donc d'optimiser, les différents paramètres d'ionisation et de fragmentation pour chacun des ions utilisés en mode de quantification, ce qui assure une sensibilité optimale. En effet, après fragmentation, les différents ions et fragments peuvent être identifiés en mode balayage (scan) ou servir à la mesure de la concentration des espèces chimiques dans l'effluent chromatographique en mode d'ions sélectionnés (SIM) : généralement un ion de quantification, majoritaire, et deux ions de confirmation par molécule étudiée. La figure 2 présente un schéma global d'ionisation, de fragmentation et de sélection des ions dans un filtre de masse quadripolaire.

\section{Les sources d'ionisation chimique à pression atmosphérique (APCI)}

Dans une source APCI, l'effluent chromatographique est nébulisé et désolvaté par vaporisation à travers un tube en quartz chauffé, ce qui provoque dans le même temps une ionisation douce (principe de la source thermospray, aujourd'hui abandonnée). Une ionisation chimique plus intense peut être obtenue à l'aide d'une décharge électrique (ou décharge corona) dans la phase vapeur. Ce sont alors essentiellement les molécules d'eau et de solvant qui sont ionisées, puis qui transmettent leur charge aux molécules à doser (figure 3).

Les sources APCI se distinguent des sources electrospray par l'ionisation de molécules plus apolaires, leur compatibilité avec des débits de phases mobiles élevés (jusqu'à $1 \mathrm{~mL} / \mathrm{min}$ ) et leur moindre sensibilité au phénomène de suppression d'ions lié à la matrice.

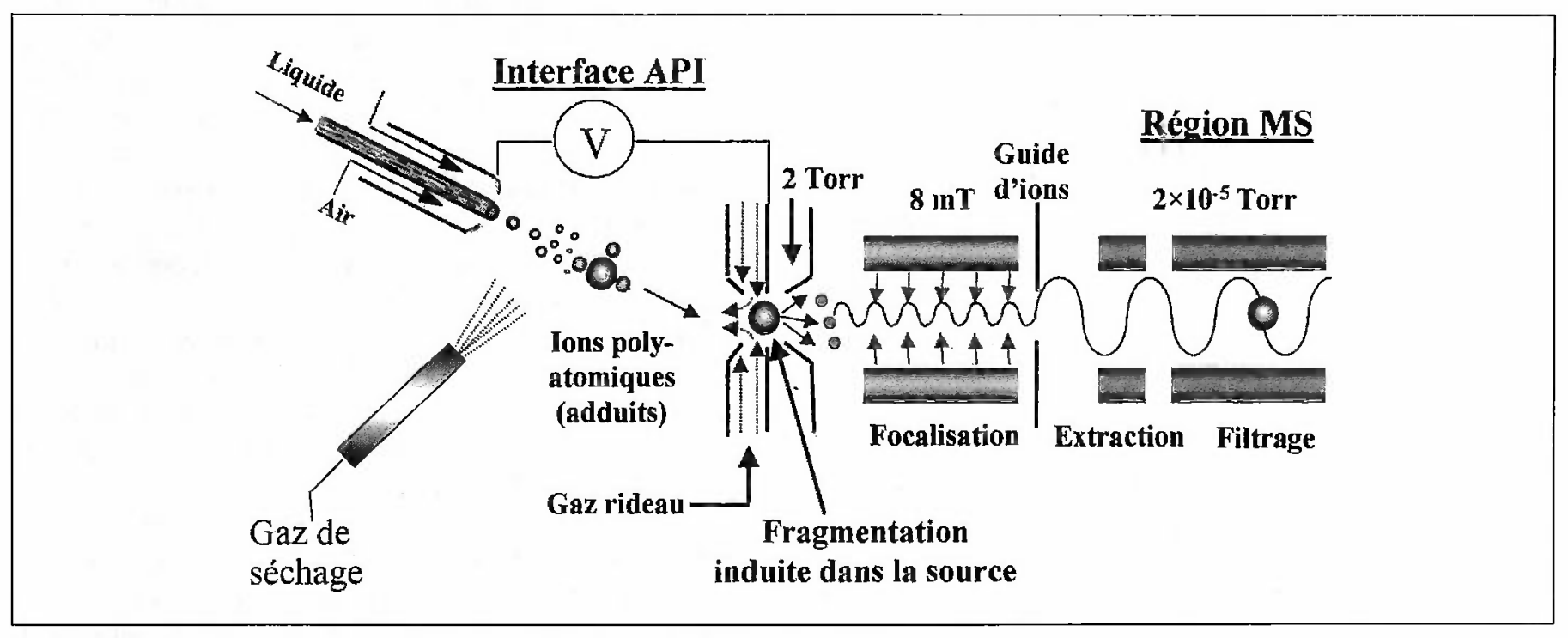

Figure 2 : Principe de la LC-MS avec source électrospray, collision induite dans la source et sélection d'ions. 


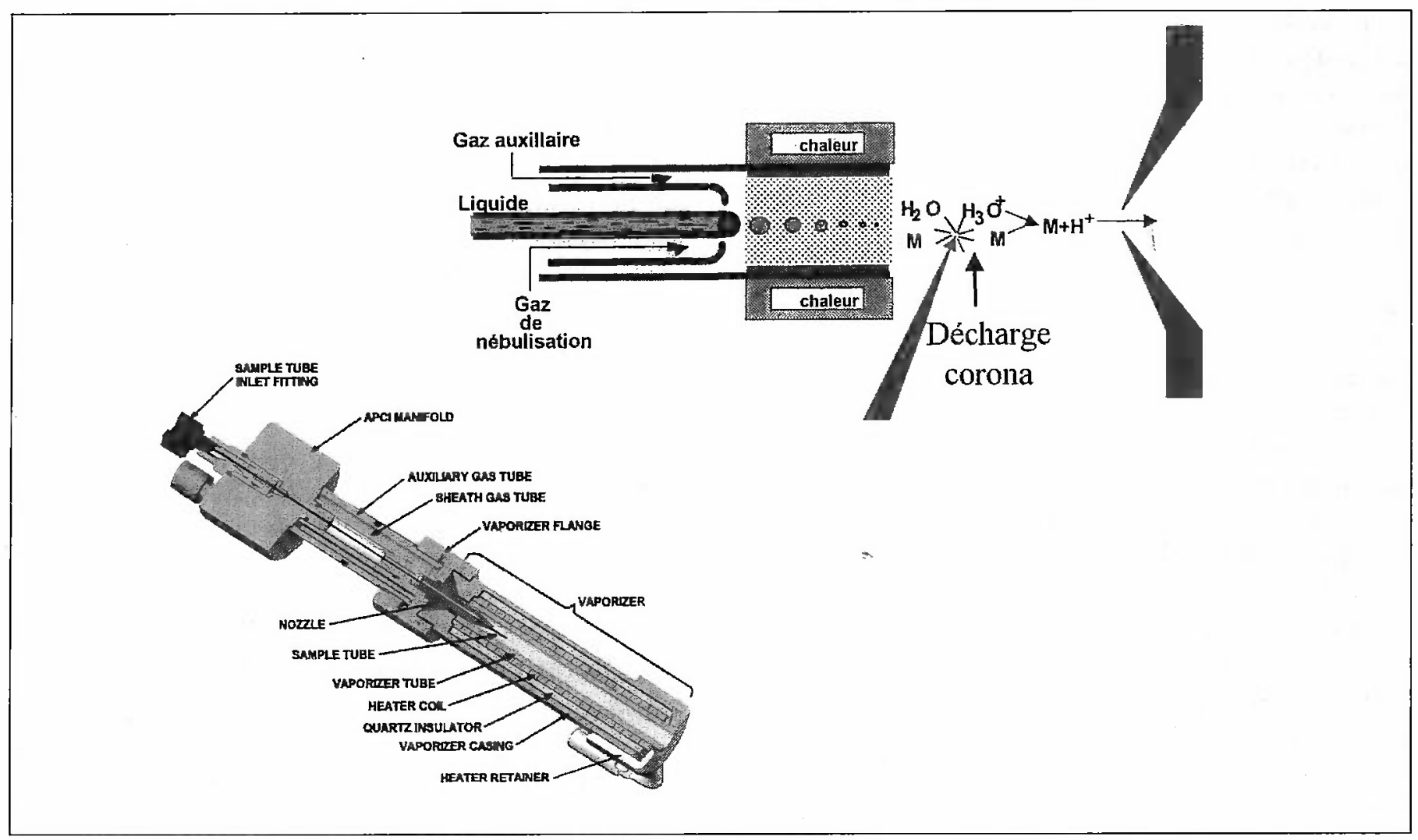

Figure 3 : Vue éclatée [d'après (9)] et schéma de principe d'une source APCI.

\section{Les sources de photo-ionisation à pres- sion atmosphérique (APPI), ou photo- spray}

Les sources photospray différent des sources APCI par le mode d'ionisation du solvant : la décharge corona est ici remplacée par une lampe UV qui permet d'ioniser une molécule dopante photosensible, rajoutée à la phase mobile. Cette molécule dopante transfère elle-même sa charge aux molécules à doser, soit directement soit en ionisant d'abord les molécules de solvant. Une molécule dopante classiquement utilisée est le toluène.

Cette source d'ionisation bénéficie d'un très faible bruit de fond chimique, contrairement à l'APCI. Elle convient particulièrement aux molécules très apolaires, pour leșquelles sa sensibilité est supérieure à celle de l'APCI ou de l'electrospray, mais elle est limitée à des molécules de 1000 à 2000 daltons. La nébulisation assistée par la chaleur peut induire, comme pour l'APCI, une décomposition des molécules thermolabiles. Son champ d'application se rapproche donc de celui du couplage chromatographie en phase gazeuse spectrométrie de masse. Les exemples classiques d'applications d'intérêt toxicologique concernent les stéroïdes naturels ou exogènes, les hydrocarbures polyaromatiques, les vitamines apolaires, etc.

A noter enfin que l'utilisation systématique d'une molécule dopante alourdit l'étape chromatographique dans la mesure où sa concentration doit rester constante, y com- pris si un gradient de phase mobile est appliqué, et où sa présence peut interférer sur la séparation chromatographique, en particulier lorsqu'il s'agit de toluène.

\section{Les cellules de collision \\ Principe de la spectrométrie de masse en tandem de quadripôles (MS/MS)}

Les sources d'ionisation à pression atmosphérique provoquant une ionisation douce, sans fragmentation, et donc avec une information structurale limitée, la spectrométrie de masse en tandem utilise une cellule de collision pour fragmenter les ions sélectionnés dans le premier filtre quadripolaire. Cette cellule de collision comporte souvent elle-même un quadripôle permettant de focaliser les ions ; elle est remplie d'un gaz neutre (généralement de l'argon, parfois de l'azote) à travers lequel les ions sont accélérés pour provoquer une fragmentation par collisions. Les fragments ainsi produits sont admis dans un deuxième filtre de masse dans lequel ils peuvent être analysés séquentiellement en mode balayage ou sélectionnés en mode de quantification (figure 4). Il existe d'autres modes MS/MS, perte de neutre et recherche d'ions parents, essentiellement utilisés pour l'identification structurale. De multiples variantes existent sur la base ou en dehors de ce schéma classique. Différentes configurations de cellules de collision sont étudiées dans les paragraphes suivants. 


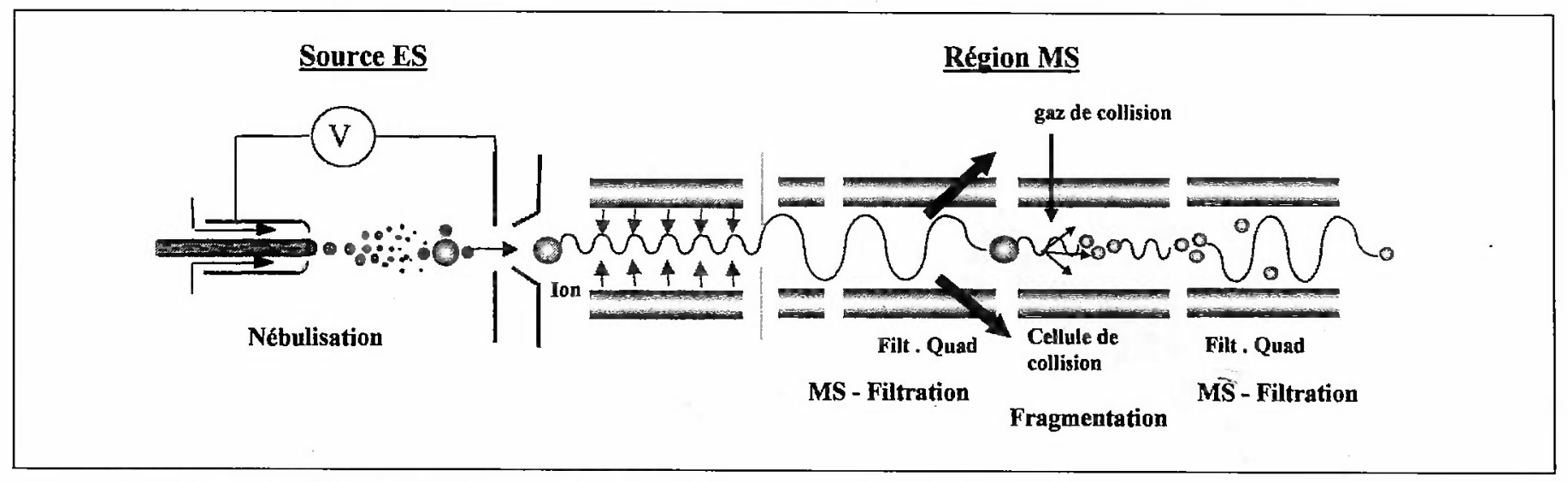

Figure 4 : Principe de la LC-ES-MS/MS quadripolaire.

\section{Solutions techniques des constructeurs}

Chez Thermo-Electron, la cellule de collision des spectromètres de masse en tandem de la série TSQQuantum décrit un quart de cercle (9), tandis que dans le système Varian $1200 \mathrm{~L}$, elle décrit un demi-cercle (10). Le but de ces deux configurations est d'éviter le passage de molécules neutres vers le deuxième filtre quadripolaire pour réduire le bruit de fond chimique parvenant au détecteur. Ces configurations présentent également l'intérêt d'un encombrement réduit et de l'utilisation d'une seule pompe turbomoléculaire à deux étages pour évacuer les deux filtres quadripolaires, contrairement aux systèmes classiques qui nécessitent généralement deux pompes.

Chez Applied-Biosystems/Sciex (8), la cellule LINAC (linearly accelerated ion) est un quadripôle dans lequel deux barres opposées sont convergentes et les deux autres barres opposées divergentes (figure 5). L'intérêt est de créer un gradient électrique entre l'entrée et la sortie de cette cellule de collision, permettant d'évacuer plus rapidement les ions générés et d'éviter un effet de traînage d'un ion parent sur le suivant, appelé effet cross-talk.

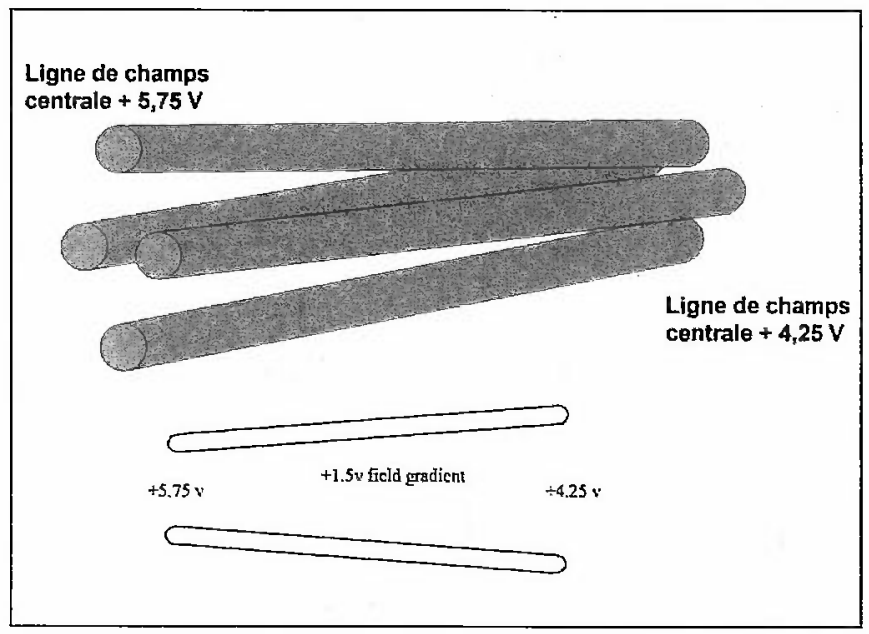

Figure 5 : Cellule LINAC ("linearly accelerated ions"), Applied-Biosystems/Sciex [d'après (8)].
Chez Waters (11), l'évacuation de la cellule de collision est encore plus rapide grâce à la technologie TWave réalisée par une succession de dizaines de lentilles ioniques délimitant la source de collision, parcourues par un train d'ondes électriques qui chassent les ions produits devant elles (figure 6). Couplé à la chromatographie à ultra-haute pression (UPLC), ce système permet de conserver une bonne résolution malgré les pics chromatographiques très fins obtenus avec des séparations de moins d'une minute.

\section{Les filtres de masse}

\section{Les filtres quadripolaires}

La théorie des filtres de masse quadripolaires repose sur l'assemblage de 4 barreaux parallèles de section hyperbolique. En réalité, la plupart des quadripôles fabriqués, aussi bien pour les couplages GS-MS que LC-MS, sont des montages de barreaux cylindriques, ce qui conduit à une perte de résolution en masse. La société Thermo-Electron (9) propose des quadripôles de section hyperbolique (HyperquadTM) qui permettent d'obtenir des pics de masse mieux résolus (largeur à mi-hauteur de 0,2 ou de $0,1 \mathrm{U}$ ) avec une transmission satisfaisante des ions (figure 7).

La Société Applied-Biosystems-Sciex a été la première à proposer un nouveau concept : la trappe d'ions quadripolaire (8), consistant à utiliser le deuxième filtre de masse quadripolaire comme une trappe d'ions, par fermeture de l'entrée et de la sortie par des barrières magnétiques et confinement des ions au centre du quadripôle. Les ions ainsi piégés peuvent être accumulés pendant un certain temps chromatographique et éjectés séquentiellement vers le détecteur, sans perte (figure 8). La résultante est une augmentation de la sensibilité dans le mode balayage d'ions fils (product ion scan ou daughter ion scan), potentiellement utile pour le screening d'inconnus en toxicologie $(12,13)$. Par ailleurs, 


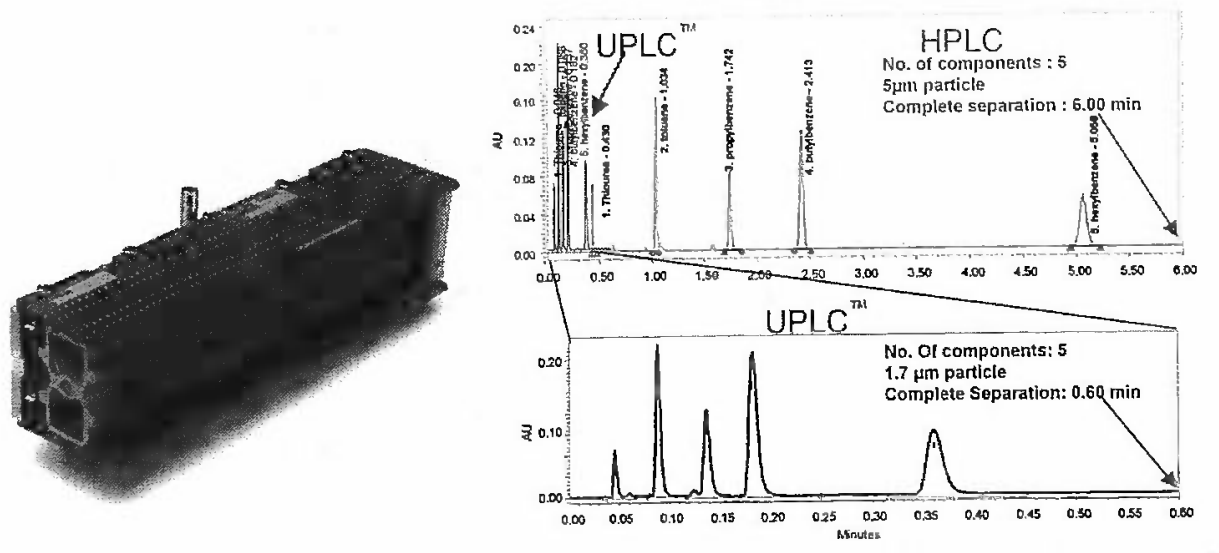

Figure 6 : Technologie de cellule de collision "T-Wave" (Waters) et couplage à la chromatographie ultra-haute pression (UPLC) [d'après (11)].

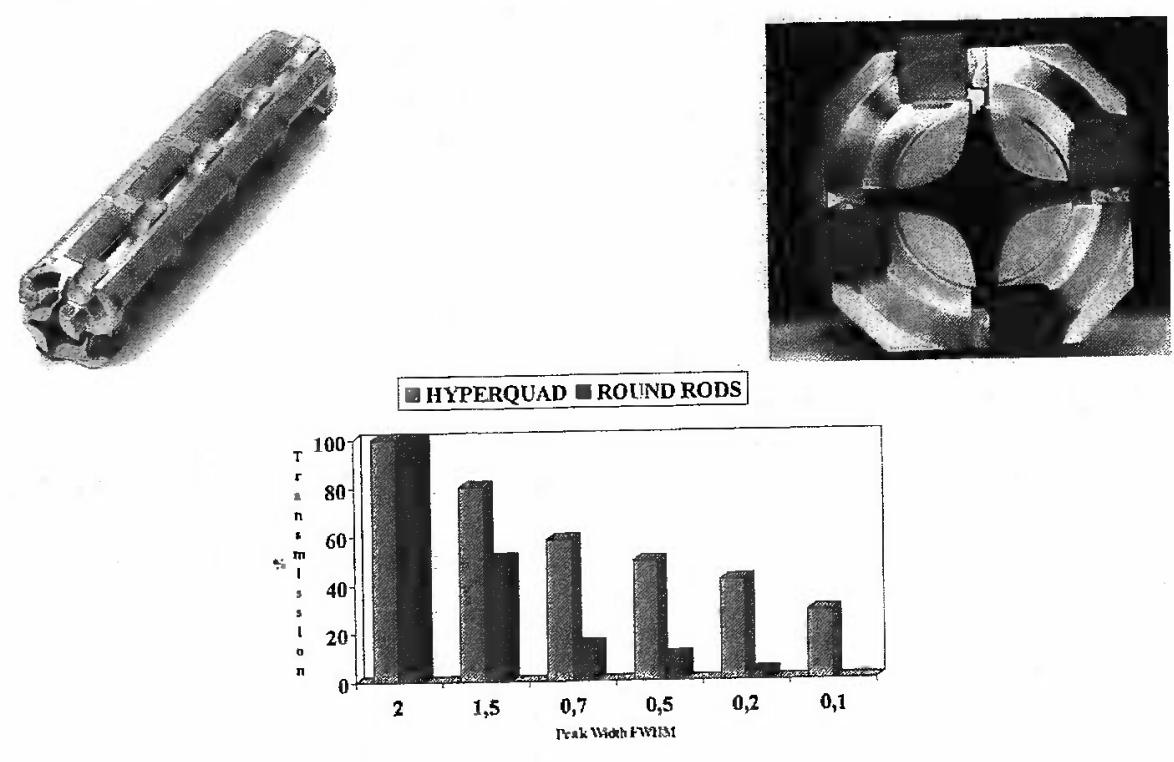

Figure 7 : Hyperquads (série TSQ-QUANTUM, Therno-Electron) et précision en masse [d'après (9)].

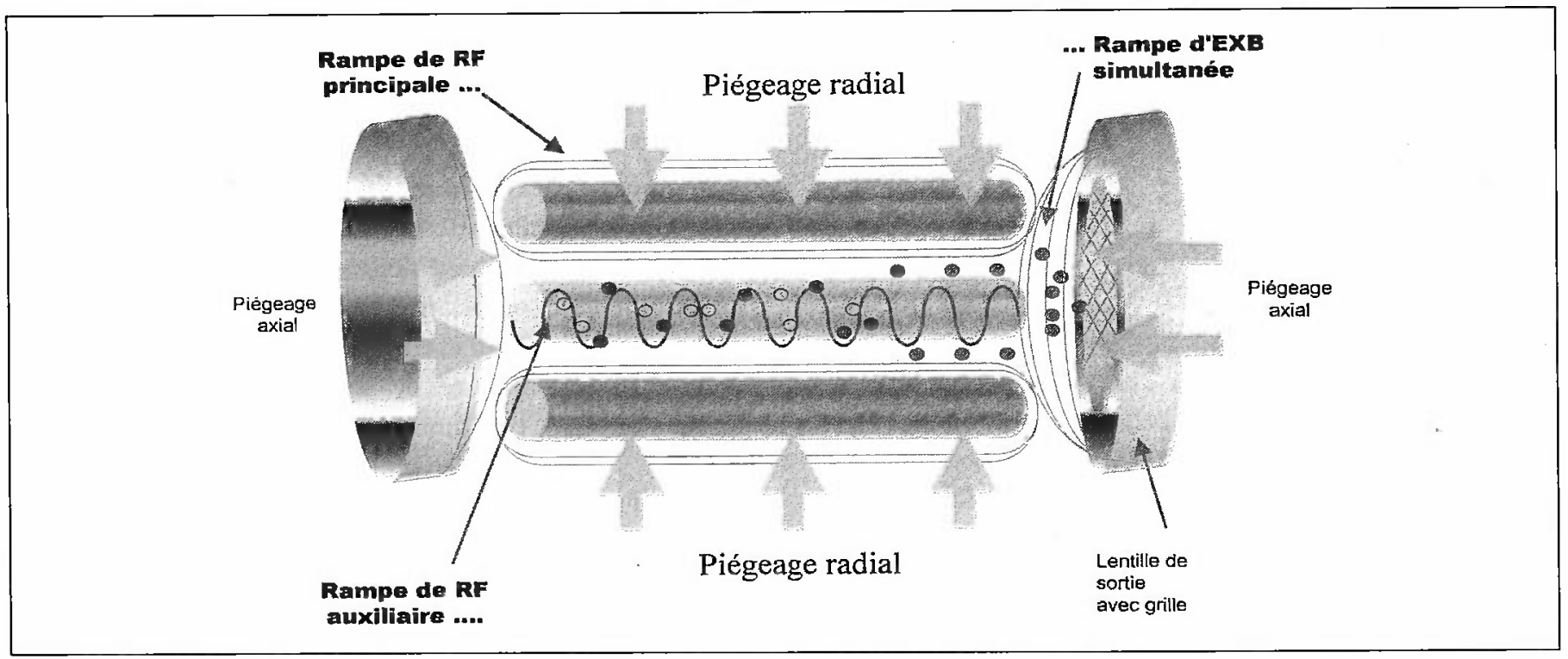

Figure 8 : Piégeage et éjection des ions dans la trappe d'ion quadripolaire d'un appareil MS/MS QTRAP AppliedBiosystems/Sciex [d'après (8)]. 
les ions accumulés dans la trappe peuvent être à nouveau fragmentés par résonance, permettant ainsi une analyse $\mathrm{MS}^{3}$. Un système similaire est également proposé par la Société Thermo-Electron, mais basé sur un simple quadripôle (système LTQ) (9).

\section{Couplages quadripôles - temps de vol (Q-TOF)}

Les détecteurs de masse à temps de vol offrent l'avantage d'une grande résolution et d'une bonne sensibilité (puisque tous les ions générés sont conservés pour la détection, contrairement aux filtres quadripolaires qui n'en conservent qu'un à chaque instant). Les systèmes hybrides comportant un premier filtre de masse quadripolaire permettent l'identification de molécules par sélection (éventuellement automatique) d'ions parents intenses, fragmentation et identification des formules atomiques les plus probables pour les fragments générés, en fonction de leur masse exacte. Cette technologie a connu un renouveau avec l'avènement de la protéomique, mais peut également se révéler intéressante pour des méthodes de screening d'inconnus en toxicologie (14). La Société Waters propose un tel appareil sous le nom Q-TOF ultima API (11). De son côté, la Société Agilent commercialise un spectromètre à temps de vol simple, sous le nom LC/MSD TOF (7).

\section{Couplage quadripôle à trappe d'ions linéaire - cyclotron.}

Pour les laboratoires les plus riches (c'est-à-dire essentiellement ceux faisant de la protéomique), la Société Thermo-Electron (9) a mis au point un couplage entre sa trappe d'ions linéaire LTQ et un spectromètre de masse cyclotronique avec transformée de Fourrier, de haute résolution. Il s'agit du système LTQ-FTMS, permettant d'obtenir une résolution en masse de moins d'1 ppm, le plus souvent utilisé pour déterminer la séquence en acides aminés des peptides multichargés issus d'étapes de protéolyse. L'utilité d'un tel système en toxicologie, outre le screening de composés inconnus, pourrait être l'analyse de familles complexes telle que celle des dioxines.

\section{Recommandations l'identification des sub- stances en LC-MS ET LC- MS/MS quantitatives.}

Dans la plupart des cas, les méthodes quantitatives utilisées en LC-MS et LC-MS/MS utilisent les modes d'ions sélectionnés (SIM), ou de transitions sélectionnées (SRM ou MRM). Comme en GC-MS, la spécificité de l'analyse repose alors sur la sélection de plusieurs ions ou de plusieurs transitions pour chaque analyte et sur la vérification que ces ions ou transitions sont coélués et dans un rapport d'intensité identique au standard. Pourtant, aucune directive concernant le nombre d'ions qualifiants ou les limites d'acceptabilité des rapports d'intensité n'avait été édictée pour la LCMS et la LC-MS/MS jusqu'à une époque récente. C'est en 2003 que l'Agence Mondiale Anti-Dopage a proposé les règles suivantes (15):

- au moins 3 ions diagnostiques (dont 1 ion de quantification), avec un rapport signal-sur-bruit toujours supérieur à 3 pour l'ion le moins intense ;

- rapports d'intensité entre ces ions dans des limites strictes, dépendant de leur intensité relative (tableau I). Lors d'un atelier consacré à la LC-MS au cours du congrès de l'IATDMCT à Bâle en 2003, nous avons proposé de sélectionner au moins 2 transitions par analyte en mode LC-MS/MS, en nous appuyant sur quelques exemples de transitions majoritaires identiques pour 2 molécules non apparentées (ex. amantadine et paracétamol). Ce phénomène est d'autant plus dangereux en LC-MS/MS que la séparation chromatographique est généralement réduite au minimum (16).

\section{Conclusion}

Dans tous les domaines d'application, le couplage de la chromatographie liquide à la spectrométrie de masse fait actuellement appel, dans la très grande majorité des cas, à des sources à pression atmosphérique : électrospray, APCI ou APPI. Par ailleurs, l'évolution des ventes et des gammes de produits montre que la spec-

Tableau I : Tolérance sur l'intensité relative des ions pour assurer une incertitude d'identification acceptable en LC-MS et LCMS/MS [d'après les recommandations de l'AMA (12)].

\begin{tabular}{|l|l|}
\hline Abondance relative $(\%$ du pic de base) & Tolérance \\
\hline$\geq 50 \%$ & $\pm 15 \%$ absolue (ex. $55-85 \%$ pour une abondance attendue de $70 \%)$ \\
\hline$<50 \%$ et $\geq 25 \%$ & $\pm 25 \%$ relative (ex. $30-50 \%$ pour une abondance attendue de $40 \%)$ \\
\hline$<25 \%$ & $\pm 10 \%$ absolue (ex. $10-30 \%$ pour une abondance attendue de $20 \%)$ \\
\hline
\end{tabular}


trométrie de masse en tandem devient la règle, aux dépens de la spectrométrie de masse simple. En toxicologie, les tandems de filtres quadripolaires sont actuellement les plus utilisés, permettant aussi bien l'identification que la quantification des molécules, mais des systèmes avec une meilleure résolution en masse pourraient se révéler utiles pour les procédures de screening, d'identification de composés inconnus ou les études de métabolisme.

Pour l'heure, le premier critère de choix d'un système (en dehors du prix) reste probablement sa sensibilité, dans la mesure où la LC-MS est utilisée en toxicologie pour la recherche et le dosage de molécules faiblement concentrées (buprénorphine, LSD, benzodiazépines, neuroleptiques, etc.). Au-delà des progrès techniques en cours et à venir, qui devraient ouvrir encore de nouvelles perspectives, de bonnes pratiques d'utilisation de ces systèmes et des critères stricts de validation des techniques doivent être appliqués pour assurer la fiabilité des analyses. En effet, la sensibilité et la spécificité intrinsèques des couplages LC-MS et LC-MS/MS ne garantissent pas à elles seules l'absence d'erreurs, d'autant que leur utilisation est généralement accompagnée d'une simplification des étapes d'extraction et de séparation chromatographique.

\section{Références}

1. Niessen, W. M. A. Advances in instrumentation in liquid chromatography-mass spectrometry and related liquidintroduction techniques. J. Chromatogr. A 1998 ; 794 : 407-35.

2. Hoja H., Marquet P., Verneuil B., Lotfi H., Penicaut B., Lachatre G. Applications of liquid chromatography-mass spectrometry in analytical toxicology : a review. J. Anal. Toxicol. $1997 ; 21(2)$ : 116-26.

3. Maurer H. Liquid chromatography-mass spectrometry in forensic and clinical toxicology. J. Chromatogr. B 1998 ; $713: 3-25$

4. Marquet P., Lachatre G. Liquid chromatography-mass spectrometry : potential in forensic and clinical toxicology. J. Chromatogr. B Biomed. Sci. Appl. $1999 ; 733$ (1-2): 93-118.

5. Van Bocxlaer J.F., Clauwaert K.M., Lambert W.E., et al.. Liquid chromatography-mass spectrometry in forensic toxicology. Mass Spectrom. Rev. 2000 ; 19 : 165-214

6. Marquet P. Progress of liquid chromatography-mass spectrometry in clinical and forensic toxicology. Ther. Drug Monit. 2002 ; 24(2) : 255-76.

7. Site Internet de la Société Agilent. http:/www.home.agilent.com (consulté le 28/12/04).

8. Site Internet de la Société Applied-Biosystems. http:/www.appliedbiosystems.com (consultéle 28/12/04).

9. Site Internet de la Société Thermo-Electron. http:/www.thermo.com (consulté le 28/12/04).

10. Site Internet de la Société Varian. http:/www.varianinc.com (consulté le 28/12/04).

11. Site Internet de la Société Waters. http:/www.waters.com (consulté le 28/12/04).

12.Marquet P., Saint-Marcoux F., Gamble T.N., Leblanc J.C. Comparison of a preliminary procedure for the general unknown screening of drugs and toxic compounds using a quadrupole-linear ion-trap mass spectrometer with a liquid chromatography-mass spectrometry reference technique. J. Chromatogr. B Analyt. Technol. Biomed. Life Sci. 2003 ; 789(1) : 9-18.

13.Jansen R., Lachâtre G., Marquet P. LC-MS/MS systematic toxicological analysis : comparison of MS/MS spectra obtained with different instruments and settings. Clin. Biochem. Clin. Biochem. 2005 ; 38(4) : 362-72.

14. Decaestecker T.N., Clauwaert K.M., Van Bocxlaer J.F., Lambert W.E., Van den Eeckhout E.G., Van Peteghem C.H., De Leenheer A.P. Evaluation of automated single mass spectrometry to tandem mass spectrometry function switching for comprehensive drug profiling analysis using a quadrupole time-of-flight mass spectrometer. Rapid Commun Mass Spectrom. 2000 ; 14(19) : 1787-92.

15. Site Internet de l'Agence Mondiale Antidopage. http:/www.wada-ama.org (consulté le 28/12/04).

16. Marquet P. Atelier "LC-MS in clinical toxicology". 8 8 me Congrès International de Suivi Thérapeutique Pharmacologique et Toxicologie Clinique (IATDMCT), 7-11 Septembre 2003, Bâle, Suisse. 